Opt i mal anount of monocyt e chemoat t r act ant pr ot ei $n-1$ enhances ant i turror ef fect $s$ of sui ci de gene ther apy agai nst hepat ocel I ul ar car ci noma by $\mathrm{M}$ macrophage act i vat i on

\begin{tabular}{|l|l|}
\hline 著者 & $\begin{array}{l}\text { Tsuchi yana Tonøya, Nakantt o Yasunar i, Sakai } \\
\text { Yoshi o, Mukai da Naof um, Kaneko Shui chi }\end{array}$ \\
\hline $\begin{array}{l}\text { j our nal or } \\
\text { publ i cat i on ti t l e }\end{array}$ & Cancer Sci ence \\
\hline vol une & 99 \\
\hline number & 10 \\
\hline page r ange & $2075-2082$ \\
\hline year & $2008-01-01$ \\
\hline URL & ht t p: //hdl . handl e. net /2297/45593 \\
\hline
\end{tabular}




\title{
Optimal amount of monocyte chemoattractant protein-1 enhances antitumor effects of suicide gene therapy against hepatocellular carcinoma by M1 macrophage activation
}

\author{
Tomoya Tsuchiyama, ${ }^{1}$ Yasunari Nakamoto, ${ }^{1}$ Yoshio Sakai,, ${ }^{1}$ Naofumi Mukaida² and Shuichi Kaneko ${ }^{1,3}$

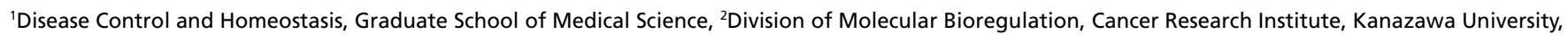 \\ 13-1 Takara-machi, Kanazawa 920-8641, Japan
}

(Received April 10, 2008/Revised June 18, 2008/Accepted June 27, 2008/Online publication October 9, 2008)

Suicide gene therapy combined with chemokines provides significant antitumor efficacy. Coexpression of suicide gene and monocyte chemoattractant protein-1 (MCP-1) increases antitumor effects in murine models of hepatocellular carcinoma (HCC) and colon cancer. However, it is unclear whether the doses administered achieved the maximum antitumor effects. We evaluated antitumor effects of various amounts of recombinant adenovirus vector ( $\mathrm{rAd}$ ) expressing MCP-1 in the presence of a suicide gene in a murine model of HCC. HCC cells were transplanted subcutaneously into BALB/c nude mice, and transduced with a fixed amount of Ad-tk harboring the suicide gene, HSV-tk, and various doses of Ad-MCP1 harboring MCP-1 (ratios of 1:1, 0.1:1, and 0.01:1 relative to Ad-tk). Growth of primary tumors was suppressed when treated with Ad-tk plus Ad-MCP1 (1:1 and $1: 0.1)$ as compared with Ad-tk alone. The antitumor effects against tumor rechallenge tended to be high in the Ad-tk plus AdMCP1 group (1:0.1). The effects were dependent on production of Th1 type-cytokines. Delivery of an optimal amount of rAd expressing MCP-1 enhanced the antitumor effects of suicide gene therapy against HCC by M1 macrophage activation, suggesting that this is a plausible form of cancer gene therapy to prevent HCC progression and recurrence. (Cancer Sci 2008; 99: 2075-2082)

ancer gene therapy using combinations of various genes, such as suicide and cytokine genes, to enhance tumor regression therapy is widely used.(1,2) Previously, we reported that the coexpression of herpes simplex virus thymidine kinase (HSV-tk) and monocyte chemoattractant protein-1 (MCP-1) showed enhanced antitumor effects in models of hepatocellular carcinoma $(\mathrm{HCC})^{(3)}$ and colon cancer, ${ }^{(4)}$ and these antitumor effects were dependent on the activation of macrophages. ${ }^{(3)} \mathrm{MCP}-$ 1 is a chemokine that regulates the recruitment of monocytes/ macrophages to inflammatory sites and tumor tissues as well as their activation, including lysosomal enzyme release and tumoricidal activity, ${ }^{(5)}$ and is functional in both mice and humans. ${ }^{(6)}$ However, MCP-1 was reported to be destructive in some tumor models, ${ }^{(6,7)}$ but protective in others. ${ }^{(8)}$ Monocytes/macrophages recruited by MCP-1 have dual functions in that they can prevent the establishment and spread of tumor cells, ${ }^{(6)}$ and simultaneously support tumor growth and dissemination. ${ }^{(8)}$ This ambivalent relationship reflects the elevated functional plasticity of macrophages, which are able to express different functional programs in response to different microenvironment signals, as exemplified in the M1 (classical)-M2 (alternative or non-classical) paradigm of macrophage polarization. ${ }^{(9)}$

On the other hand, although double infection methods are used to enhance antitumor effects in cancer gene therapy, significant antitumor effects have been reported in some studies, ${ }^{(4,10)}$ but not in others. ${ }^{(11,12)}$ Moreover, it is not clear how the antitumor effects are affected by differences in the doses administered. In the present study, various amounts of recombinant adenovirus vector (rAd) expressing the $M C P-1$ gene were delivered into cells along with the same amount of HSV-tk to determine the optimal dosage of MCP-1 for induction of stronger antitumor effects in double infection methods. Furthermore, we also examined the involvement of macrophage immune responses in these effects. Here, we demonstrated that treatment with the 1:0.1 ratio of Ad-HSV-tk (Ad-tk) plus Ad-MCP1 tended to exert antitumor immunity, suggesting that there may be an optimal amount of Ad-MCP1 in suicide gene therapy. In addition, it is possible that the antitumor responses seen in the HSV-tk plus MCP-1 system were associated with increased Th1 (T helper 1)-type cytokine production by activated M1 macrophage. These findings will be of value in cancer gene therapy.

\section{Materials and Methods}

Recombinant adenoviruses. rAds harboring the human MCP-1 (Ad-MCP1), HSV-tk (Ad-tk), and lacZ (Ad-lacZ), and driven by the CAG promoter were prepared, purified, and titrated according to the protocols supplied by the manufacturer (Takara Bio, Shiga, Japan), as described. ${ }^{(13)}$ The rAds were purified on cesium gradients and their titers were determined by the $50 \%$ tissue culture infectious dose $\left(\mathrm{TCID}_{50}\right)$.

Cell lines and culture. The human HCC cell line Huh7 and the mouse HCC cell line BNL 1ME A.7R.1 (BNL) were cultured in Dulbecco's minimal essential medium (Gibco, Long Island, NY, USA) supplemented with $10 \%$ heat-inactivated fetal bovine serum (Gibco).

Enzyme-linked immunosorbent assay (ELISA) for MCP-1. Aliquots of $1 \times 10^{5}$ Huh 7 cells were seeded in $1.0 \mathrm{~mL}$ of culture media in 24-well tissue culture plates. Twenty-four h later, the cells were infected with each rAd at a multiplicity of infection (MOI) of 10 , and the medium was collected $48 \mathrm{~h}$ later. On the other hand, in some experiments, ganciclovir (GCV; Tanabe Pharmaceutical Drug, Tokyo, Japan) $(10 \mu \mathrm{g} / \mathrm{mL})$ was added $72 \mathrm{~h}$ later, and the medium was collected and replaced with the same volume of fresh medium every $24 \mathrm{~h}$. The concentration of MCP-1 in the medium collected from each well was determined by ELISA as described. ${ }^{(14)}$

In vivo studies in nude mice. The following investigations were performed in accordance with the guidelines of our Institutional Animal Care and Use Committee. Six-week-old male athymic

${ }^{3}$ To whom correspondence should be addressed. E-mail: skaneko@m-kanazawa.jp 
nude mice (BALB/cA Jcl-nu; CLEA Japan, Tokyo, Japan) were injected subcutaneously with $1 \times 10^{7} \mathrm{HuH} 7$ cells at the both sides of the flank on day 0 . On days 3 and $4,1 \times 10^{7}$ TCID $_{50}$ $(100 \mu \mathrm{L})$ of Ad-tk, Ad-lacZ, or Ad-tk $\left(1 \times 10^{7} \mathrm{TCID}_{50}\right.$, fixed dose) plus Ad-MCP1 (1, 0.1, 0.01, or $0.001 \times 10^{7}{ }^{50}$ TCID $_{50}$, changed dose) were injected into the tumor. Then, $75 \mathrm{mg} / \mathrm{kg}$ of GCV was administered into the peritoneal cavity daily for the next 5 consecutive days (day 5-9), and tumor size was measured every 3 days. Tumor volumes were calculated using the formula:

$$
\underline{(\text { longest diameter }) \times(\text { shortest diameter })^{2}}
$$

2

Gene expression analysis (real-time reverse transcription-polymerase chain reaction [RT-PCR]). Total RNA was extracted from tumor tissues or spleens resected after treatment of the tumor with each rAd, using a Total Cellular RNA Isolation Kit (Ambion, St. Austin, TX, USA), in accordance with the manufacturer's protocol. The RNA was reverse transcribed with a TaqMan reverse transcription reagent kit (PE Applied Biosystems, Foster City, CA, USA) using random hexamer primers. Gene expression was analyzed by real-time RT-PCR using TaqMan Universal Master Mix on an ABI PRISM 7900 Sequence Detection System (PE Applied Biosystems). The PCR primer pairs for mouse interleukin (IL)-10, IL-12, IL-18, IFN- $\gamma$, VEGF, and 18S rRNA were obtained from the TaqMan assay reagent library. Data for whole samples were normalized to $18 \mathrm{~S}$ rRNA and then expressed as the fold change in mRNA expression as compared with control samples treated with phosphate-buffered saline (PBS).

Immunohistochemical analysis. Tumor tissues were resected on day 10. The tissue samples were embedded in OCT compound (Sakura Finetek, Torrance, CA, USA) and snap-frozen in liquid nitrogen. Cryostat sections of frozen tissues were fixed in cold acetone for $10 \mathrm{~min}$, followed by three rinses in PBS. To avoid non-specific staining, avidin and biotin in the tissues were blocked using a blocking kit (Vector Laboratories, Burlingame, CA, USA). The slides were subsequently incubated with antibodies (Abs) against Mac-1 (M1/70; Pharmingen, San Diego, CA, USA) for $30 \mathrm{~min}$ at room temperature. Negative controls included staining with non-specific $\mathrm{Ab}$ of the corresponding isotype and subsequent staining with secondary $\mathrm{Ab}$. The reactions were visualized using a VECTASTAIN ABC Standard Kit (Vector Laboratories), followed by counterstaining with hematoxylin.

Preparation of peritoneal exudate macrophages and assays for cytokine production in vitro. Thioglycolate-elicited murine peritoneal exudate cells were collected as described. ${ }^{(15)}$ Briefly, nude or immunocompetent mice were injected intraperitoneally with $2 \mathrm{~mL}$ each of $3 \%$ fluid thioglycolate medium (Wako Pure Chemical) and sacrificed 4 days later, followed by peritoneal lavage with $10 \mathrm{~mL}$ of cold PBS. About $90 \%$ of the collected peritoneal cells were positive for both Mac-1 (CD11b) and I-A ${ }^{\mathrm{d}}$ MHC class II as determined by staining with PE-conjugated anti-Mac-1 Ab and fluorescein-isothiocyanate (FITC)-conjugated I-A ${ }^{\mathrm{d}}$ MHC class II (AMS-32.1; Pharmingen). Huh7 cells were infected with rAds, at a MOI of 5 for $24 \mathrm{~h}$. Aliquots of $10^{5}$ macrophages were cocultured with $10^{5}$ rAd-treated Huh7 cells in $1.0 \mathrm{~mL}$ of culture media in 24-well tissue culture plates, and treated with GCV for 2 days at $37^{\circ} \mathrm{C}$. The concentrations of IL-10, IL-12, IL-18, and IFN- $\gamma$ in the media were quantified using immunoassay kits (IL-10, IL-12, IFN- $\gamma$ : Biosource International, Camarillo, CA, USA; IL-18: Medical \& Biological Laboratories, Nagoya, Japan).

Rechallenge testing in nude mice. Nude mice were injected subcutaneously with $5 \times 10^{6} \mathrm{HuH} 7$ cells on day 0 . On days 3 and 4, the subcutaneous tumors were injected with $5 \times 10^{7}$ TCID $_{50}(100 \mu \mathrm{L})$ of Ad-tk, Ad-lacZ, or Ad-tk (fixed dose) plus Ad-MCP1 (changed dose), and the mice were treated with $75 \mathrm{mg} / \mathrm{kg} \mathrm{GCV}$, injected into the peritoneal cavity, every day for the next 5 days (days 5-9). Following complete eradication of the primary tumors, the mice were subcutaneously rechallenged on day 14 with $3 \times 10^{6} \mathrm{HuH} 7$ cells at two sites, which were more than $3 \mathrm{~cm}$ apart from the primary challenge site. Two of 10 $(20 \%)$ mice treated with Ad-tk and four of $30(13.3 \%)$ treated with Ad-tk plus Ad-MCP1 did not show complete eradication of the primary tumor by the final measurement and were therefore excluded from the rechallenge experiment. Tumor sizes were measured every 4 days after the second tumor injection, and tumor volumes were calculated using the formula:

$\frac{(\text { longest diameter }) \times(\text { shortest diameter })^{2}}{2}$

Animal studies in immunocompetent mice (ex vivo, in vivo, and rechallenge). Six-week-old immunocompetent male BALB/c-jcl mice (CLEA Japan) were injected subcutaneously with $1 \times 10^{5}$ BNL cells infected with each rAd at an in vitro MOI of 5 at the both sides of the flank on day 0 , and GCV was administered intraperitoneally for the next 5 days (days 1-5). Tumor size was measured every 7 days, and tumor volume was calculated using the formula:

$$
\frac{(\text { longest diameter }) \times(\text { shortest diameter })^{2}}{2}
$$

As with the experiments on nude mice, BALB/c-jcl mice were injected subcutaneously with $1 \times 10^{5} \mathrm{BNL}$ cells at the both sides of the flank on day 0 . On days 3 and $4,5 \times 10^{5}$ TCID $_{50}(100 \mu \mathrm{L})$ of rAds were injected into the tumor. Then, GCV was administered for the next 5 days (day 5-9), and tumor size was measured every 3 days.

In another experiment, immunocompetent mice were injected subcutaneously with $1 \times 10^{5}$ BNL cells infected with each rAd at an in vitro MOI of 100 on day 0 , and GCV was administered intraperitoneally for the next 5 days (days 1-5). The primary tumors were completely eradicated in all groups. These mice were injected subcutaneously with $1 \times 10^{4}$ BNL cells on day 14 at two sites which were separate from the primary challenge sites, and the tumor sizes were measured every 7 days after the second tumor injection.

ELISA for serum IL-10, IL-12, and IL-18. Mouse sera were collected prior to injection of subcutaneous primary tumors and on day 35 after tumor injection. IL-10, IL-12, and IL-18 concentrations were measured using immunoassay kits (IL-12, Biosource International; IL-18, Medical \& Biological Laboratories).

Flow cytometry. Single-cell suspensions of splenocytes were resuspended in PBS containing $1 \%$ bovine serum albumin and $0.1 \%$ sodium azide, and incubated for $30 \mathrm{~min}$ on ice with FITC-conjugated rat antimouse-F4/80 (Serotec, Oxford, UK) and PE-conjugated rat antimouse pan natural killer (NK) cells (DX5; Pharmingen), with FITC-conjugated rat antimouse-CD3 (Pharmingen) and PE-conjugated rat antimouse CD11c (Pharmingen) or with FITC-conjugated rat antimouse-CD8 (Pharmingen) and PE-conjugated rat antimouse CD4 (Pharmingen). The cells were washed, resuspended in PBS, and analyzed using a FACScan with CellQuest software.

Statistical analysis. All results are expressed as means \pm SE. The statistical significance of differences between groups was evaluated by the Mann-Whitney $U$-test.

\section{Results}

MCP-1 production by double infection with recombinant adenoviruses in vitro. $\mathrm{MCP}-1$ expression level by Ad-MCP1 alone was high compared with double infection of Ad-lacZ plus Ad-MCP1 (Fig. 1a). The amounts of MCP-1 produced by Ad-tk plus Ad-MCP1 decreased rapidly after GCV administration due to Huh7 cell 

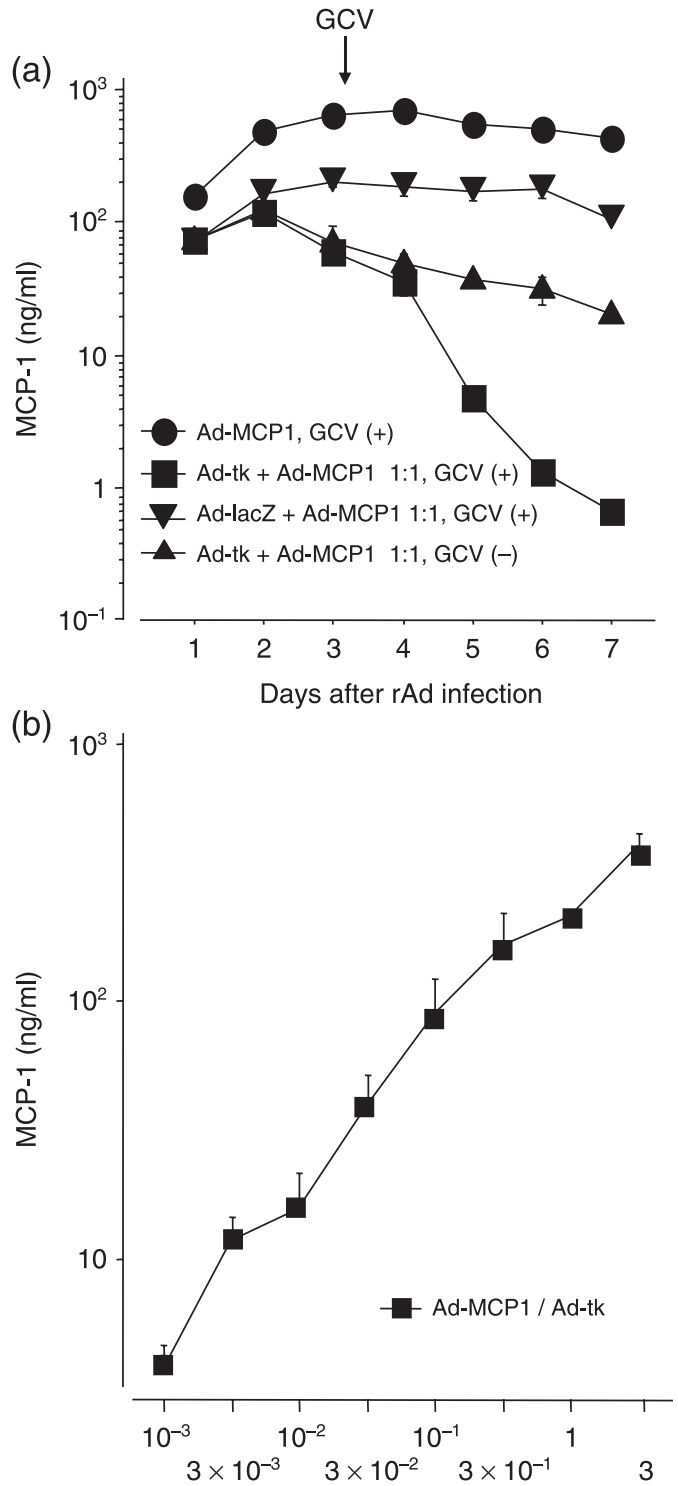

The rate of Ad-MCP1 / Ad-tk at 10 of MOI

Fig. 1. Monocyte chemoattractant protein-1 (MCP-1) production of recombinant adenoviruses in the presence of herpes simplex virus thymidine kinase (HSV-tk). Aliquots of $1 \times 10^{5}$ Huh7 cells were seeded in $1.0 \mathrm{~mL}$ of culture media in 24-well tissue culture plates. (a) Twentyfour $\mathrm{h}$ later, the cells were treated with Ad-tk plus Ad-MCP1, Ad-lacZ plus Ad-MCP1, or Ad-MCP1 at a multiplicity of infection (MOI) of 10, and treated $72 \mathrm{~h}$ later with ganciclovir (GCV) $(10 \mu \mathrm{g} / \mathrm{mL})$. Every $24 \mathrm{~h}$, the medium was collected and replaced with the same volume of fresh medium. (b) Twenty-four $h$ later, the cells were doubly infected with Ad-tk (fixed dose, at an MOI of 10) plus Ad-MCP1 (changed dose), and the medium was collected $48 \mathrm{~h}$ later. The concentrations of MCP-1 were evaluated using an immunoassay. Values are shown as the means \pm SE of duplicate experiments.

apoptosis induced by the HSV-tk/GCV system (Fig. 1a). Moreover, the amounts of MCP-1 produced by Ad-tk plus Ad-MCP1 without GCV administration were lower than those of Ad-lacZ plus Ad-MCP1, presumably due to the MCP-1 promoter interference by HSV-tk.

Next, production of MCP-1 in Huh7 cells double-infected with Ad-tk (fixed dose) plus Ad-MCP1 (changed dose) was measured. The amounts of MCP-1 were correlated with the infectious dose of Ad-MCP1 in the presence of a fixed amount of HSV-tk (Fig. 1b). (a) Subcutaneous injection with $\mathrm{HuH} 7$ cells

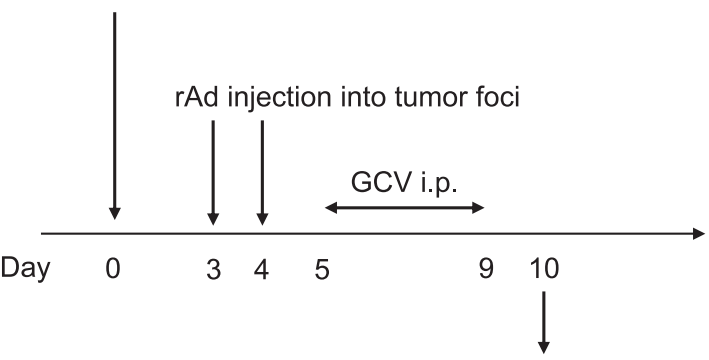

Tumor resection

(b)

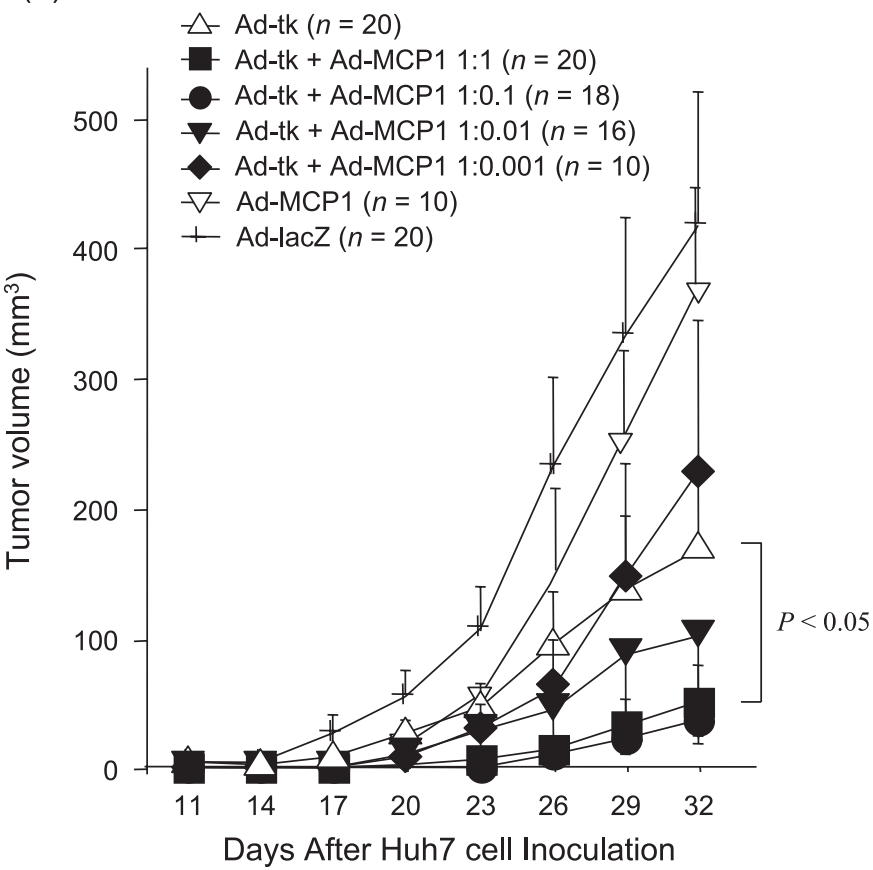

Fig. 2. The antitumor effects of the herpes simplex virus thymidine kinase (HSV-tk)/ganciclovir (GCV) system by codelivery of monocyte chemoattractant protein-1 (MCP-1) in a nude mouse model of hepatocellular carcinoma (HCC). (a) Mice were injected subcutaneously with $1 \times 10^{7}$ Huh7 cells at the both sides of the flank on day 0 . On days 3 and $4,1 \times 10^{7} \mathrm{TCID}_{50}$ of Ad-tk, Ad-tk $\left(1 \times 10^{7} \mathrm{TCID}_{50}\right.$, fixed dose) plus Ad-MCP1 $\left(1,0.1,0.01\right.$, or $0.001 \times 10^{7} \mathrm{TCID}_{50}$ changed dose), or Ad-lacZ was injected into the tumor, and the mice were injected intraperitoneally (i.p.) with $75 \mathrm{mg} / \mathrm{kg}$ of GCV every day for the next 5 days (day 5-9). (b) Tumor size was measured every 3 days. The results are shown as the means of two independent experiments.

Antitumor effects of the HSV-tk/GCV system by codelivery of the MCP-1 gene in an athymic nude mouse model of HCC. The in vivo antitumor effects of double infection with rAds were analyzed using athymic nude mice (Fig. 2a). The growth of subcutaneous tumors was markedly suppressed in animals treated with Ad-tk plus Ad-MCP1 (1:1) (tumor volume 32 days after injection, 44.4 $\left.\pm 22.5 \mathrm{~mm}^{3}\right)$ or Ad-tk plus Ad-MCP1 (1:0.1) $\left(37.4 \pm 18.6 \mathrm{~mm}^{3}\right)$, as compared to those treated with Ad-tk alone $\left(170.2 \pm 49.8 \mathrm{~mm}^{3}\right.$, $P<0.05$ ) (Fig. 2b). These observations indicated that optimal amounts of MCP-1 are needed to eradicate tumor cells in the presence of HSV-tk.

Recruitment and activation of macrophages into tumor tissues. Macrophages play important roles in both Th1- and Th2-mediated immune responses. Classical macrophage (M1 macrophages) are also a major source of IL-12 and IL-18, whereas alternative macrophages (M2 macrophages) are a source of IL-10. ${ }^{(9)}$ IL-12 

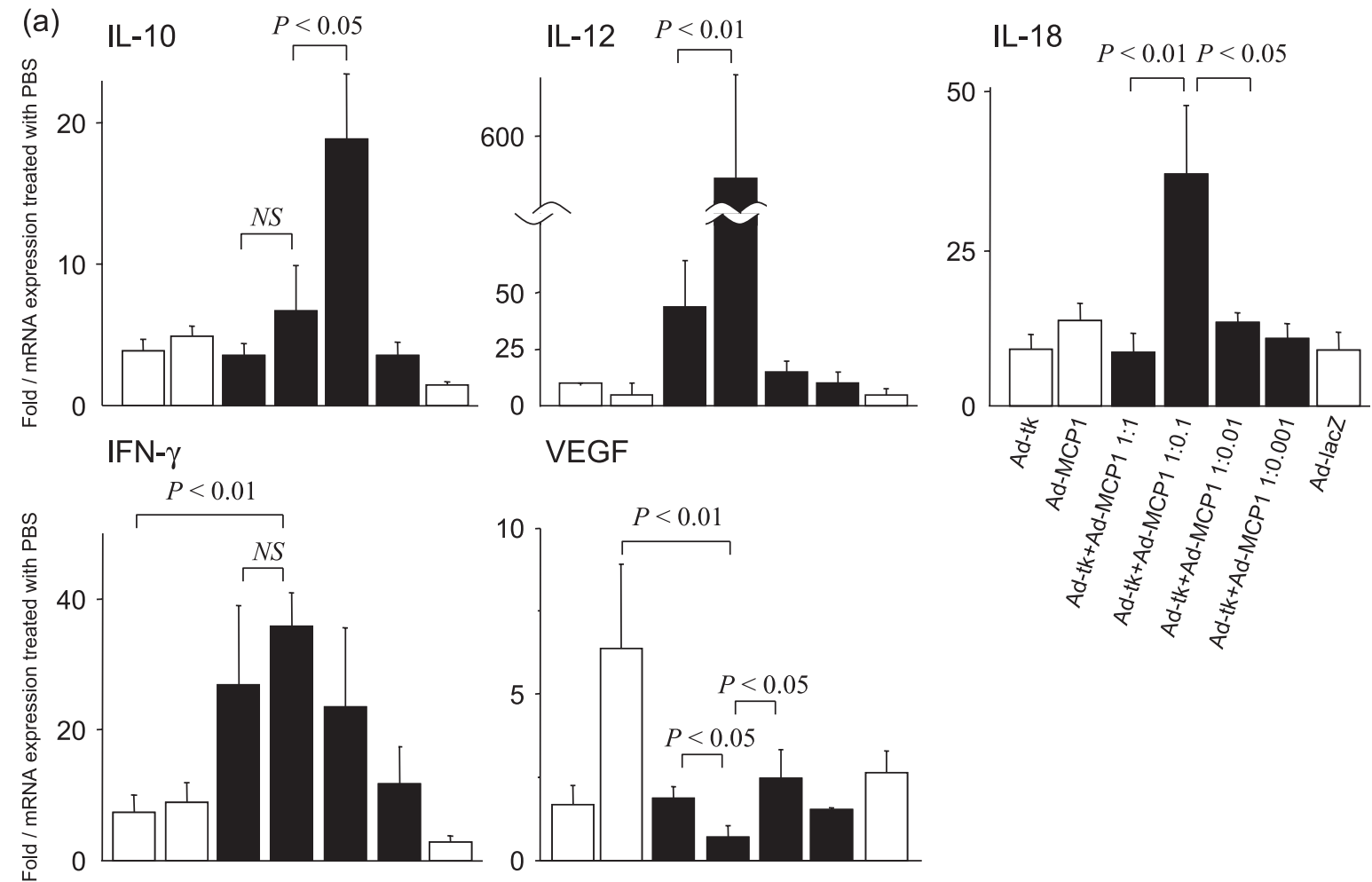

(b)
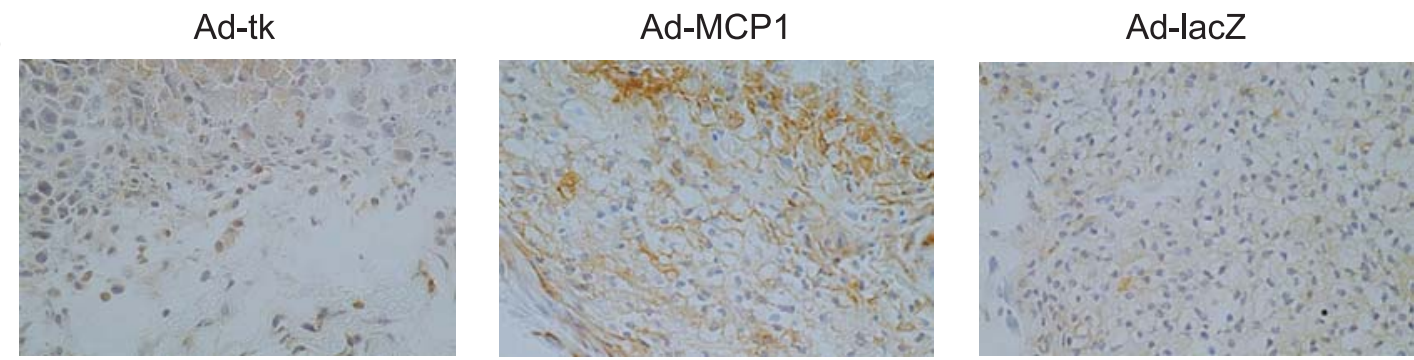

Ad-tk + Ad-MCP1 1:1

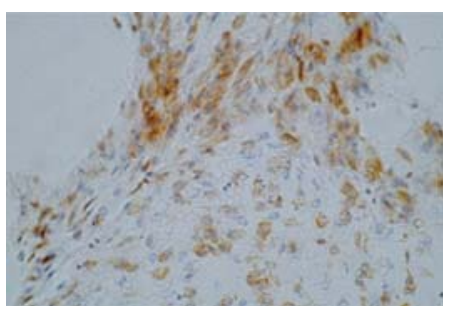

\section{Ad-tk + Ad-MCP1 1:0.1}

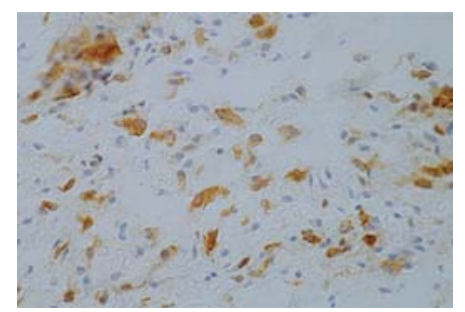

Ad-tk + Ad-MCP1 1:0.01

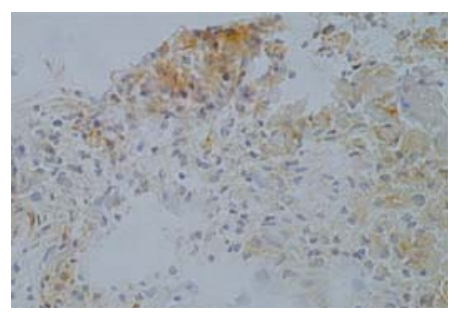

Fig. 3. Cytokine expression and macrophage recruitment in primary tumor tissues. In the experiment described in the legend to Fig. 2 , tumor tissues were resected 10 days after tumor injection. (a) Total RNA was extracted to determine cytokine mRNA levels by a real-time reverse transcription-polymerase chain reaction as described in 'Materials and Methods'. Cytokine mRNA expression was normalized to 18S rRNA and then expressed as the fold change in mRNA expression as compared with control samples treated with phosphate-buffered saline. Splenocytes treated with $0.1 \mu \mathrm{g} / \mathrm{mL}$ LPS were used as a positive control (data not shown). The results are shown as the means of two independent experiments. (b) Tumor tissues were processed for immunohistochemical analysis using anti-Mac1 antibody as described in 'Materials and Methods'. Representative results from individual animals in each group are shown here.

enhances the activities of NK cells and cytotoxic T lymphocytes (CTL), and plays a key role in the induction of Th1-type immune responses. ${ }^{(16)}$ In addition, IL-18 is a proinflammatory cytokine produced by activated macrophages, which has been shown to induce Th1 cell development and NK cell activation in combination with IL-12. ${ }^{(17)}$ In contrast, the effects of IL-10 on immune responses are mostly inhibitory. ${ }^{(18)}$ Therefore, to evaluate whether M1 macrophages recruited into tumor tissues following infection with rAds were activated, IL-10, IL-12, IL-18, IFN- $\gamma$, and VEGF expression were determined using real-time RT-PCR. IL-12 and IL-18 mRNA levels were significantly increased $(P<0.01)$, and that of IFN- $\gamma$ mRNA tended to increase in tumors treated with Ad-tk plus Ad-MCP1 (1:0.1) (Fig. 3a). In contrast, IL-10 mRNA was significantly increased in tumors treated with Ad-tk plus Ad-MCP1 (1:0.01) $(P<0.05)$ (Fig. 3a). In addition, the VEGF mRNA level was significantly increased in tumors treated with Ad-MCP1 $(P<0.01)$, and was significantly decreased in tumors treated with Ad-tk plus Ad-MCP1 (1:0.1) 

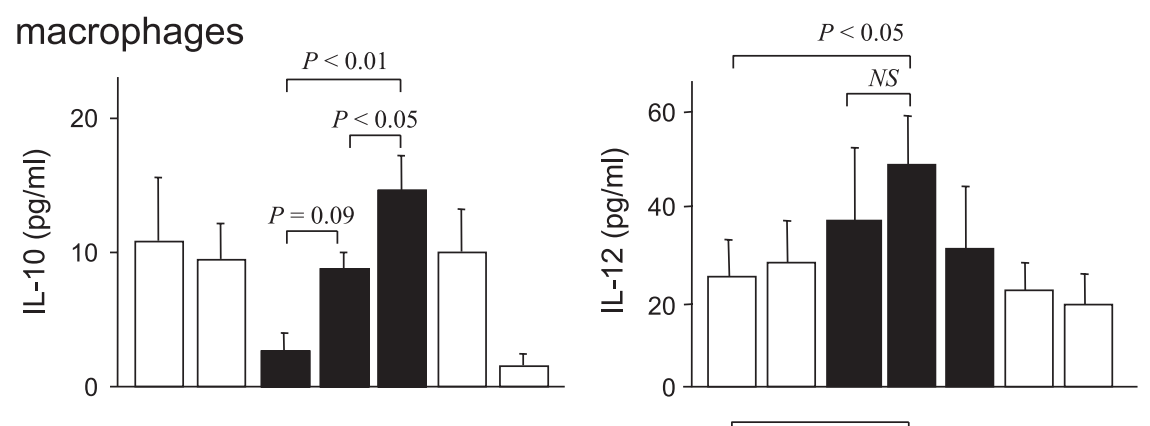

Fig. 4. Cytokine production by peritoneal macrophages cocultured with Huh7 cells infected with rAds in vitro. Huh7 cells were infected with each rAd at a multiplicity of infection (MOI) of 5 and treated with ganciclovir (GCV) for $24 \mathrm{~h}$. Aliquots of $10^{5}$ peritoneal exudate macrophages were cocultured with $10^{5}$ rAd-treated Huh7 cells for 2 days, and the concentrations of IL-10, IL-12, IL-18, and IFN- $\gamma$ in the media were evaluated by immunoassay. Values are shown as the means \pm SE of duplicate experiments.
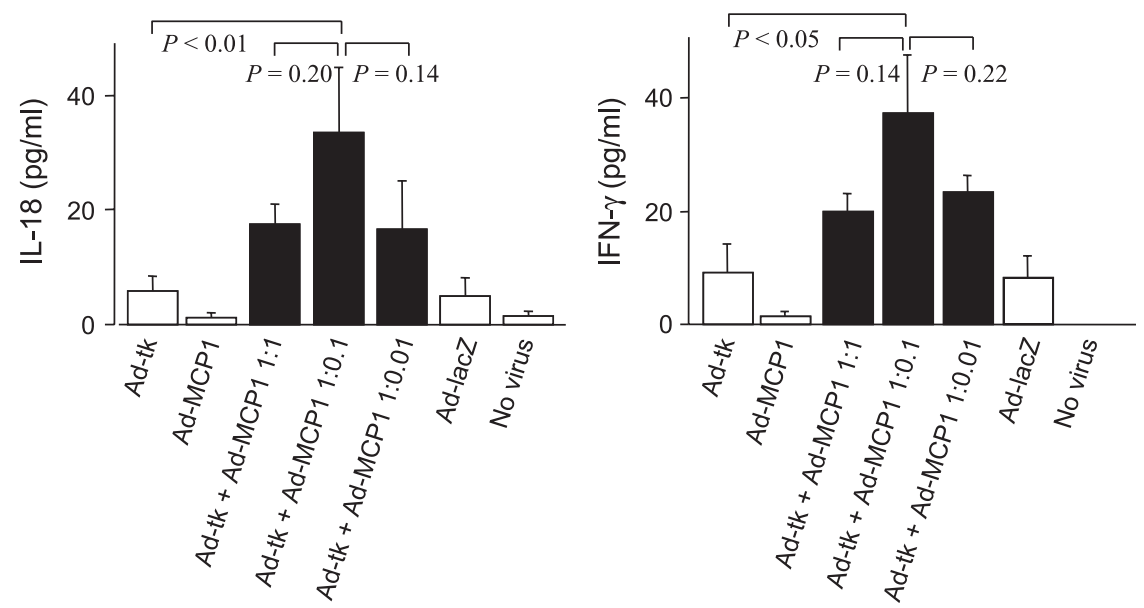

$(P<0.05)$ (Fig. 3a). Taken together, these observations indicated that M1 macrophages were highly activated when tumors were treated with the optimal dose of MCP-1 and HSV-tk.

Next, we evaluated whether there were differences in the number of macrophages recruited into tumor tissues. The number of accumulated Mac-1-positive cells in the tumors treated with Ad-tk plus Ad-MCP1 (1:0.1) was comparable to that in those treated with Ad-tk plus Ad-MCP1 (1:1) (Fig. 3b). These observations suggested that the number of recruited macrophages is of little importance to the antitumor effects.

IL-10, IL-12, IL-18, and IFN- $\gamma$ production by coculture of apoptotic Huh7 cells expressing MCP-1 and peritoneal macrophages in vitro. It was reported that adenoviral-mediated overexpression of MCP-1 differentially modulated the development of Th1 and Th2-type responses. ${ }^{(19)}$ To evaluate the differences in the immunomodulatory effects of macrophages among double infection of rAds, we measured IL-10, IL-12, IL-18, and IFN- $\gamma$ production by peritoneal exudate cells consisting mostly of macrophages, when they were cocultured with Huh7 cells infected with rAds. We found that peritoneal macrophages cocultured with Huh7 cells treated with Ad-tk plus Ad-MCP1 (1:0.1) tended to produce increased levels of IL-12, IL-18, and IFN- $\gamma$ (Fig. 4). On the other hand, the increase in amount of IL-10 in the double infection groups was inversely proportional to the dosage of MCP-1 vector (Fig. 4). These observations also suggest that the optimal dose of MCP-1 and HSV-tk may induce M1 macrophage activation.

Antitumor immunity in the rechallenge test of the HSV-tk/GCV system by codelivery of the $M C P-1$ gene. After primary subcutaneous Huh7 cells were completely eradicated with rAds, nude mice were rechallenged with Huh7 cells to evaluate antitumor immunity induced by MCP-1 plus HSV-tk. We found that the tumor regrowth was significantly suppressed when the primary tumor cells had been eradicated with Ad-tk plus Ad-MCP1 (1:0.1) as compared with Ad-tk (tumor volume 40 days after rechallenge: $123.2 \pm 77.2 \mathrm{~mm}^{3}$ vs $544.5 \pm 161.6 \mathrm{~mm}^{3}$, respectively, $P<0.05$ ) (Fig. 5). In addition, tumor regrowth tended to be low when eradicated with Ad-tk plus Ad-MCP1 (1:0.1) as compared with Ad-tk plus Ad-MCP1 (1:1) $\left(287.9 \pm 137.1 \mathrm{~mm}^{3}, P=0.18\right)$ or Ad-tk plus Ad-MCP1 (1:0.01) $\left(269.7 \pm 91.1 \mathrm{~mm}^{3}, P=0.24\right)$. Next, to evaluate immunomodulatory effects of splenocytes, we examined IFN- $\gamma$ expression using real-time RT-PCR. IFN- $\gamma$ mRNA levels were significantly increased in the spleens of nude mice treated with Ad-tk plus Ad-MCP1 (1:0.1) (Fig. 5b). Consistent with our previous findings, ${ }^{(20)}$ we observed increased numbers of NK cells in the spleen and rechallenged tumor tissues when treated with the 1:0.1 ratio of Ad-tk and Ad-MCP1 (data not shown). These results indicated that the optimal dose of MCP-1 induced beneficial antitumor immunity in the presence of HSV-tk.

Antitumor effects and immunity of the HSV-tk/GCV system plus MCP-1 treatment in an immunocompetent mouse model of HCC. There is no CTL in athymic nude mice. Therefore, to evaluate the Th1 cytokine response in the syngeneic system, the ex vivo antitumor effects of double infection with rAds were analyzed using immunocompetent BALB/c-jcl mice. The growth of subcutaneous tumors treated with Ad-tk plus Ad-MCP1 (1:1, $1: 0.1)$ was comparable to that in nude mice $(P<0.01)$, excluding the group in which the dose of MCP-1 was small $(1: 0.01)$ (Fig. 6a).

In the next experiment, after the BALB/c mice developed tumor mass following the injection with non-infected BNL cells, we infected the resultant tumors with Ad-tk plus Ad-MCP1 and treated the animals with GCV using the same procedures as the experiments with nude mice. Tumor growth was apparently retarded when treated with Ad-tk plus Ad-MCP1 $(1: 1)(P<0.05)$ and $(1: 0.1)(P<0.01)$ as compared with Ad-tk alone (Fig. 6b). However, the treatments failed to eradicate tumors completely, probably because the infection efficiency was not sufficient under these conditions.

Thus, we chose the ex vivo infection experiment in the immunocompetent mouse model, to evaluate whether rechallenged tumors could be rejected in the mice in which the primary tumors had been completely eradicated. The immunocompetent mice were rechallenged with BNL 1ME A.7R.1 (BNL) cells 

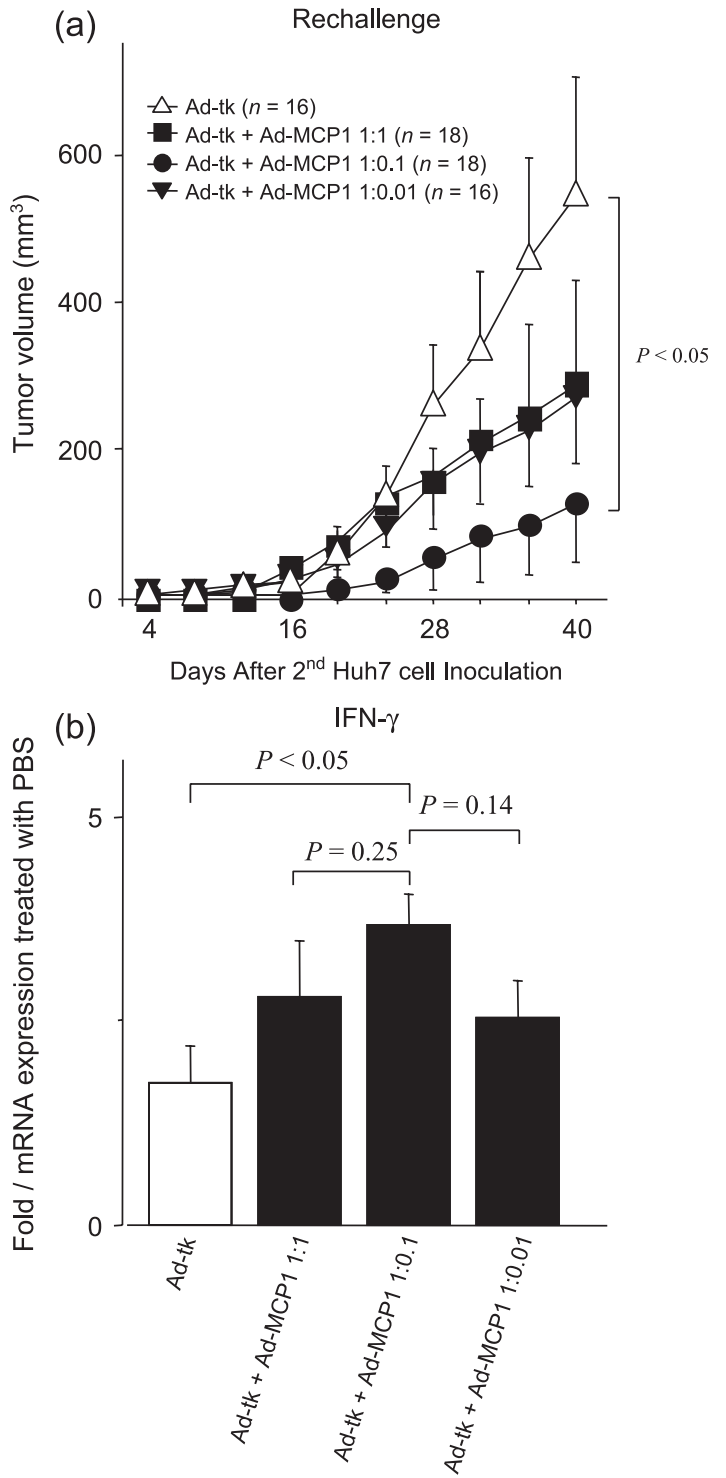

Fig. 5. Nude mice were injected subcutaneously with $5 \times 10^{6}$ Huh7 cells on day 0 . On days 3 and $4,5 \times 10^{7} \mathrm{TCID}_{50}$ of Ad-tk $(100 \mu \mathrm{L})$, Ad-tk $\left(1 \times 10^{7} \mathrm{TCID}_{50}\right.$, fixed dose) plus Ad-MCP1 $\left(1,0.1,0.01\right.$, or $0.001 \times 10^{7}$ $\mathrm{TCID}_{50}$, changed dose), or Ad-lacZ was injected into the tumor, and the mice were injected intraperitoneally with $75 \mathrm{mg} / \mathrm{kg}$ of ganciclovir (GCV) every day for the next 5 days (day 5-9). Following complete eradication of the primary tumors, the mice were subcutaneously rechallenged on day 14 with $3 \times 10^{6}$ Huh7 cells at the other sites. (a) Tumor size was measured every 4 days. (b) In another series of experiments, the spleen was resected on day 16 after tumor injection, and IFN- $\gamma$, mRNA levels were evaluated using real-time reverse transcription-polymerase chain reaction. The results are shown as the means of two independent experiments. PBS, phosphate-buffered saline.

using the same procedures as in the experiments with nude mice. Although the inhibition of tumor regrowth was significantly lower when they had been eradicated with Ad-tk plus Ad-MCP1 (1:0.1) as compared with Ad-tk (tumor volume 42 days after rechallenge: $263.9 \pm 87.8 \mathrm{~mm}^{3}$ vs $669.5 \pm 158.3 \mathrm{~mm}^{3}$, respectively, $P<0.05)$, it also tended to be lower when the primary tumor cells had been eradicated with Ad-tk plus Ad-MCP1 (1:1) (tumor volume 42 days after rechallenge: $372.5 \pm 157.8 \mathrm{~mm}^{3}$ ) (Fig. 6c), similar to the observations in athymic nude mice.

Next, we examined IL-10, IL-12, and IL-18 production on day 35 after tumor injection. Serum concentrations of IL-12 and IL-18 tended to be higher in mice treated with Ad-tk plus
Ad-MCP1 (1:0.1) as compared with those treated with Ad-tk, Ad-tk plus Ad-MCP1 (1:1), or Ad-tk plus Ad-MCP1 (1:0.01) (Fig. 6d). In contrast, the increase in amount of serum IL-10 in the double infection groups was inversely proportional to the dosage of MCP-1 (Fig. 6d). These observations were consistent with the data shown in Figs 3 and 4.

Finally, to monitor the activation state of innate and acquired immunity in extrahepatic lymphoid organs, we examined the numbers of immune cells in the spleen on day 35 after tumor injection by FACS analysis. The numbers of F4/80-positive cells tended to be higher in the Ad-tk plus Ad-MCP1 (1:1) and Ad-tk plus Ad-MCP1 (1:0.1) groups, and the numbers of DX5-positive cells tended to be higher in the Ad-tk plus Ad-MCP1 (1:0.1) group (Fig. 6e). Furthermore, the numbers of CD3-, CD4-, and CD8-positive cells were increased in the immunocompetent mice in the order of Ad-tk plus Ad-MCP1 (1:0.1), Ad-tk plus Ad-MCP1 (1:1), and Ad-tk plus Ad-MCP1 (1:0.01) (Fig. 6e). Taken together, these results confirmed that treatment with Ad-tk plus Ad-MCP1 (1:0.1) resulted in the development of beneficial antitumor immunity in both immunodeficient and immunocompetent animals.

\section{Discussion}

$\mathrm{HCC}$ is one of the most common cancer-related causes of death, and is resistant to anticancer drugs. ${ }^{(21)}$ Although gene therapy has the potential to more effectively induce tumor cell death as compared to conventional treatment, there have been no previous comparisons with regard to the optimal doses of vectors in combined gene therapy. Whereas the amounts of MCP-1 were correlated with the infectious dose of Ad-MCP1 in the presence of a fixed dose of Ad-tk, MCP-1 expression level in the presence of intracellular HSV-tk was inhibited as compared with coinfection with Ad-MCP1 plus Ad-lacZ, suggesting that HSV-tk may influence the efficiency of transcription in the transformed cells. In addition, MCP-1 expression level by Ad-MCP1 alone was high as compared with double infection with Ad-MCP1 plus AdlacZ, which was probably due to promoter interference. On the other hand, our previous study demonstrated that the levels of HSV-tk expression in cells cotransfected with Ad-tk plus AdMCP-1 were comparable to those of Ad-tk alone or Ad-tk plus Ad-lacZ. ${ }^{(3)}$ The effect of a bicistronic rAd expressing mainly HSV-tk was clearly stronger than that of a bicistronic rAd expressing mainly MCP-1. Therefore, we proposed that the

Fig. 6. Antitumor effects of the herpes simplex virus thymidine kinase (HSV-tk)/ganciclovir (GCV) system by codelivery of monocyte chemoattractant protein-1 (MCP-1) in an immunocompetent mouse model of HCC. (a) Mice were injected subcutaneously with $1 \times 10^{5} \mathrm{BNL}$ cells infected with each rAd at an in vitro multiplicity of infection (MOI) of 5 at the both sides of the flank on day 0. GCV was administered intraperitoneally for the next 5 days (days $1-5$ ), and tumor size was measured every 7 days. (b) BALB/c-jcl mice were injected subcutaneously with $1 \times 10^{5} \mathrm{BNL}$ cells at the both sides of the flank on day 0 . On days 3 and $4,5 \times 10^{5} \operatorname{TCID}_{50}(100 \mu \mathrm{L})$ of rAds were injected into the tumor. Then, GCV was administered for the next 5 days (day 5-9), and tumor size was measured every 3 days. (c) BALB/c-jcl mice were injected subcutaneously with $1 \times 10^{5} \mathrm{BNL}$ cells infected with each rAd at an in vitro $\mathrm{MOI}$ of 100 on day 0 , and GCV was administered intraperitoneally for the next 5 days (days 1-5). The primary tumors were completely eradicated in all groups. These mice were injected subcutaneously with $1 \times 10^{4} \mathrm{BNL}$ cells at other sites on day 14 , and the tumor sizes were measured every 7 days after the second tumor injection. (d) Mouse sera were collected prior to subcutaneous injection of primary tumor cells (untreated), after treatment of the tumor with each rAd, and 2 days after rechallenge with $\mathrm{HuH7}$ cells, and IL-12 and IL-18 concentrations were measured using immunoassay kits. (e) The spleen was removed to obtain single cell suspensions on day 35 after tumor injection. Surface expression of DX5, F4/80, CD3, CD4, CD8, and CD11c in cell populations obtained from the spleen were assessed by FACS. The results are representative of two independent experiments. 
(a)
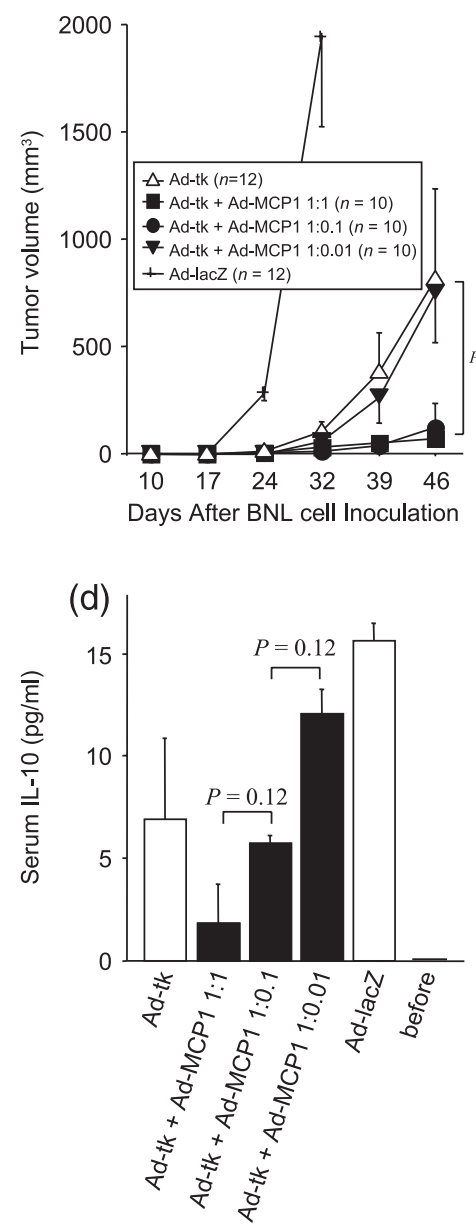

(e)
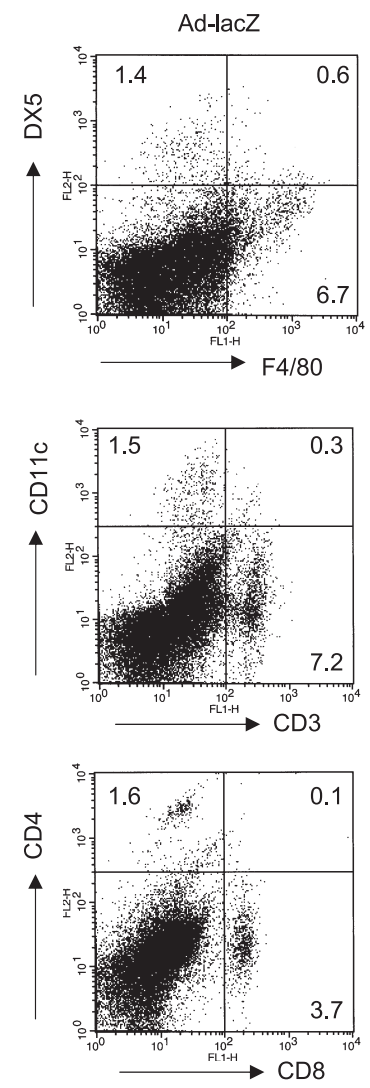

(b)
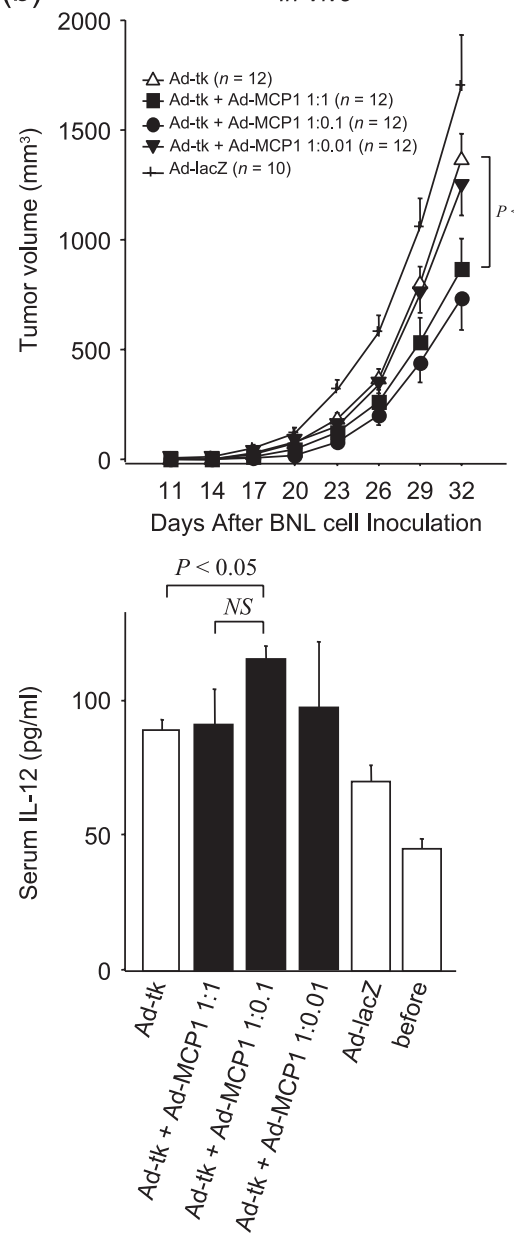

(c) Rechallenge

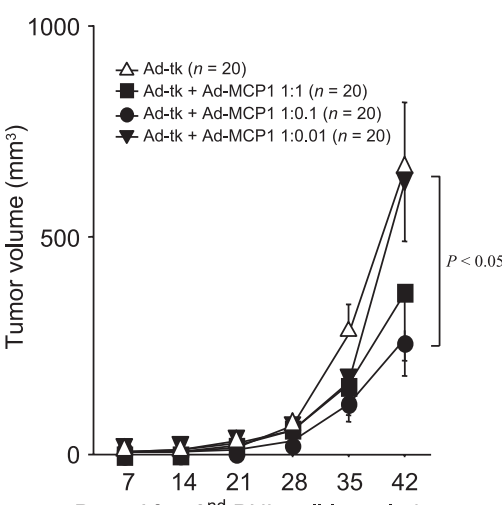

Days After $2^{\text {nd }}$ BNL cell Inoculation

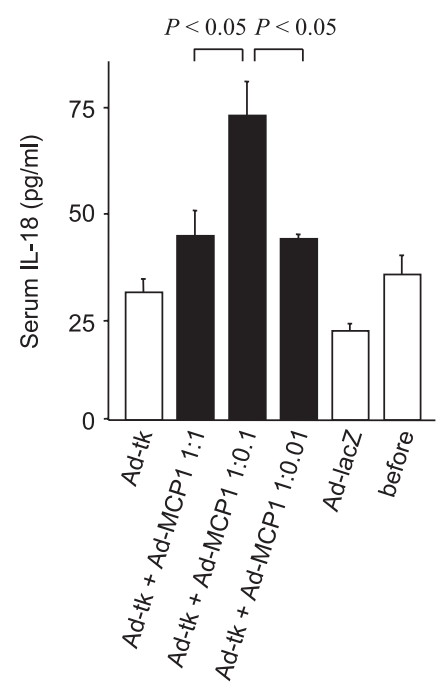

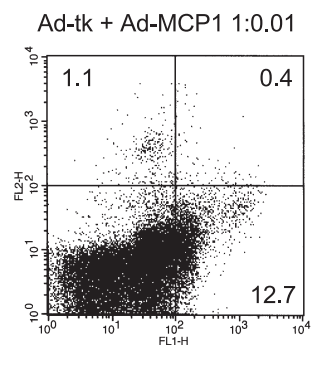

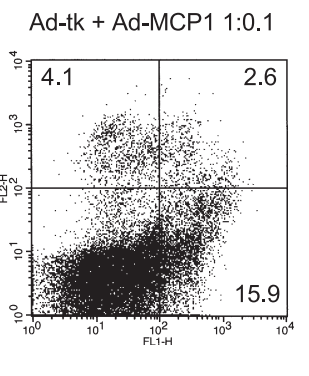

Ad-tk + Ad-MCP1 1:1
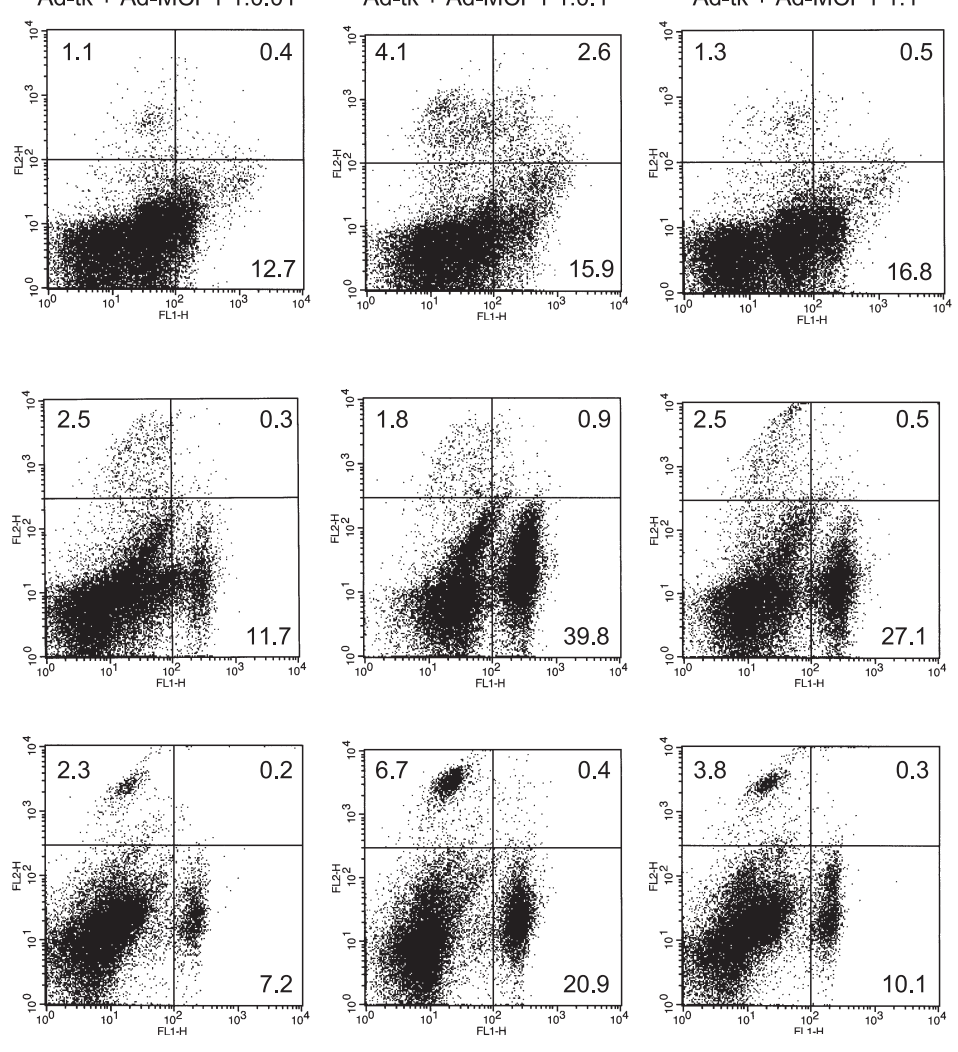
HSV-tk/GCV system should mainly be used and the use of MCP-1 was supported in our experimental models, although their efficiencies may vary depending on the nature of the cell type and reporter genes used.(22)

Th1 cytokine expression levels in tumors treated with Ad-tk plus Ad-MCP1 (1:0.1) were higher than those treated with Adtk plus Ad-MCP1 (1:1) or (1:0.01). Moreover, macrophages produced large amounts of Th1 cytokines when cocultured with apoptotic HCC cells induced by Ad-tk plus Ad-MCP1 (1:0.1). In contrast, whereas the amounts of $\mathrm{Th} 2$ cytokines were relatively high in Ad-tk plus Ad-MCP1 (1:0.01), they were low in Ad-tk plus Ad-MCP1 (1:1). There were almost no differences in the number of macrophages among the tumors treated with various combinations of HSV-tk and MCP-1. Therefore, the types of activated macrophages may be important rather than the numbers recruited and activated. The ratio of IL-12 to IL-10 can be used as a simple metric to classify activated macrophages into two categories, M1 or M2. ${ }^{(23,24)}$ M1 macrophages are potent effector cells that kill microorganisms and tumor cells and produce large amounts of proinflammatory cytokines, particularly IL-12. In contrast, M2 macrophages, a producer of IL-10, tune inflammatory responses and adaptive Th1 immunity, scavenge debris, and promote angiogenesis, tissue remodeling, and repair. The M1/M2 dichotomy of macrophage polarization can elicit both anti- and pro-tumoral activities. ${ }^{(25)}$

MCP-1 is known to facilitate tumor growth under different conditions, probably by promoting angiogenesis. ${ }^{(8)}$ In the present study, the VEGF expression levels in tumors treated with Ad-tk

\section{References}

1 Okada H, Miyamura K, Itoh T et al. Gene therapy against an experimental glioma using adeno-associated virus vectors. Gene Ther 1996; 3: 957-64.

2 Coll JL, Mesnil M, Lefebvre MF, Lancon A, Favrot MC. Long-term survival of immunocompetent rats with intraperitoneal colon carcinoma tumors using herpes simplex thymidine kinase/ganciclovir and IL-2 treatments. Gene Ther 1997; 4: 1160-6.

3 Tsuchiyama T, Kaneko S, Nakamoto Y et al. Enhanced antitumor effects of a bicistronic adenovirus vector expressing both herpes simplex virus thymidine kinase and monocyte chemoattractant protein-1 against hepatocellular carcinoma. Cancer Gene Ther 2003; 10: 260-9.

4 Kagaya T, Nakamoto Y, Sakai Y et al. Monocyte chemoattractant protein-1 gene delivery enhances antitumor effects of herpes simplex virus thymidine kinase/ganciclovir system in a model of colon cancer. Cancer Gene Ther 2006; 13: 357-66.

5 Matsushima K, Larsen CG, DuBois GC, Oppenheim JJ. Purification and characterization of a novel monocyte chemotactic and activating factor produced by a human myelomonocytic cell line. J Exp Med 1989; 169: 1485-90.

6 Rollins BJ, Sunday ME. Suppression of tumor formation in vivo by expression of the JE gene in malignant cells. Mol Cell Biol 1991; 11: 3125-31.

7 Nokihara H, Yanagawa H, Nishioka Y et al. Natural killer cell-dependent suppression of systemic spread of human lung adenocarcinoma cells by monocyte chemoattractant protein-1 gene transfection in severe combined immunodeficient mice. Cancer Res 2000; 60: 7002-7.

8 Ueno T, Toi M, Saji H et al. Significance of macrophage chemoattractant protein-1 in macrophage recruitment, angiogenesis, and survival in human breast cancer. Clin Cancer Res 2000; 6: 3282-9.

9 Mantovani A, Sozzani S, Locati M, Allavena P, Sica A. Macrophage polarization: tumor-associated macrophages as a paradigm for polarized M2 mononuclear phagocytes. Trends Immunol 2002; 23: 549-55.

10 Kijima T, Osaki T, Nishino K et al. Application of the Cre recombinase/loxP system further enhances antitumor effects in cell type-specific gene therapy against carcinoembryonic antigen-producing cancer. Cancer Res 1999; 59: 4906-11.

11 Freund CT, Sutton MA, Dang T, Contant CF, Rowley D, Lerner SP. Adenovirus-mediated combination suicide and cytokine gene therapy for bladder cancer. Anticancer Res 2000; 20: 1359-65.

12 Sakai Y, Kaneko S, Sato Y et al. Gene therapy for hepatocellular carcinoma using two recombinant adenovirus vectors with alpha-fetoprotein promoter and Cre/lox P system. J Virol Meth 2001; 92: 5-17.

13 Sato Y, Tanaka K, Lee G et al. Enhanced and specific gene expression via tissue-specific production of Cre recombinase using adenovirus vector. Biochem Biophys Res Commun 1998; 244: 455-62.

14 Sakai Y, Kaneko S, Nakamoto Y, Kagaya T, Mukaida N, Kobayashi K Enhanced anti-tumor effects of herpes simplex virus thymidine kinase/ plus Ad-MCP1 (1:0.1) were low as compared with those treated with Ad-MCP1 alone or Ad-tk plus Ad-MCP1 (1:1 and 1:0.01). A previous study indicated that monocyte recruitment is dependent on the level of MCP-1 secreted by the tumor cells and that the effects of monocyte infiltration on tumor growth are dependent on their levels of infiltration. ${ }^{(26)} \mathrm{MCP}-1$ secreted by apoptotic Huh7 cells may have recruited macrophages more efficiently to these apoptotic cells, thereby resulting in a greater deleterious effect on tumor formation. Therefore, we propose that it is necessary to set the appropriate dosages of the two vectors in the HSV-tk plus MCP-1 system.

Recently, we found that the HSV-tk/GCV system, together with delivery of MCP-1, eradicated HCC and exerted prolonged antitumor effects by activating macrophages and NK cells. ${ }^{(20)}$ In this study, the antitumor immunity increased in mice treated with Ad-tk plus Ad-MCP1 (1:0.1). Several investigators have reported that dying HSV-tk-modified cells released soluble factors, including cytokines. ${ }^{(27,28)}$ These factors could in turn affect the tumor microenvironment, leading to necrosis and inflammation, infiltration of immune cells, up-regulation of costimulatory molecules, and generation of an antitumorigenic immune responses. ${ }^{(28,29)}$ In this immunotherapeutically favorable setting, the optimal dose of MCP-1 with HSV-tk inside the same cell may stimulate tumor-specific immune-mediated cell killing. Consequently, the delivery of an optimal amount of rAd expressing MCP-1 enhanced the antitumor effects of the HSV-tk/GCV system in a model of HCC, and the effects were related to the balance of Th1 and Th2-type cytokines.

ganciclovir system by codelivering monocyte chemoattractant protein-1 in hepatocellular carcinoma. Cancer Gene Ther 2001; 8: 695-704.

15 Kawaguchi T, Suematsu M, Koizumi HM et al. Activation of macrophage function by intraperitoneal administration of the streptococcal antitumor agent OK-432. Immunopharmacology 1983; 6: 177-89.

16 Lamont AG, Adorini L. IL-12: a key cytokine in immune regulation. Immunol Today 1996; 17: 214-7.

17 Okamura H, Kashiwamura S, Tsutsui H, Yoshimoto T, Nakanishi K. Regulation of interferon-gamma production by IL-12 and IL-18. Curr Opin Immunol 1998; 10: 259-64.

18 Moore KW, de Waal Malefyt R, Coffman RL, O'Garra A. Interleukin-10 and the interleukin-10 receptor. Annu Rev Immunol 2001; 19: 683-765.

19 Matsukawa A, Lukacs NW, Standiford TJ, Chensue SW, Kunkel SL. Adenoviral-mediated overexpression of monocyte chemoattractant protein-1 differentially alters the development of Th1 and Th2 type responses in vivo. J Immunol 2000; 164: 1699-704.

20 Tsuchiyama T, Nakamoto Y, Sakai Y et al. Prolonged, NK cell-mediated antitumor effects of suicide gene therapy combined with monocyte chemoattractant protein-1 against hepatocellular carcinoma. J Immunol 2007; 178: $574-83$.

21 Okita K. Management of hepatocellular carcinoma in Japan. J Gastroenterol 2006; 41: 100-6.

22 Mizuguchi H, Xu Z, Ishii-Watabe A, Uchida E, Hayakawa T. IRES dependent second gene expression is significantly lower than cap-dependent first gene expression in a bicistronic vector. Mol Ther 2000; 1: 376-82.

23 Mantovani A, Sica A, Sozzani S, Allavena P, Vecchi A, Locati M. The chemokine system in diverse forms of macrophage activation and polarization. Trends Immunol 2004; 25: 677-86.

24 Biswas SK, Gangi L, Paul S et al. A distinct and unique transcriptional program expressed by tumor-associated macrophages (defective NF-kappaB and enhanced IRF-3/STAT1 activation). Blood 2006; 107: 2112-22.

25 Mantovani A, Bottazzi B, Colotta F, Sozzani S, Ruco L. The origin and function of tumor-associated macrophages. Immunol Today 1992; 13: 265-70.

26 Nesbit M, Schaider H, Miller TH, Herlyn M. Low-level monocyte chemoattractant protein-1 stimulation of monocytes leads to tumor formation in nontumorigenic melanoma cells. J Immunol 2001; 166: 6483-90.

27 Barba D, Hardin J, Sadelain M, Gage FH. Development of anti-tumor immunity following thymidine kinase-mediated killing of experimental brain tumors. Proc Natl Acad Sci USA 1994; 91: 4348-52.

28 Vile RG, Castleden S, Marshall J, Camplejohn R, Upton C, Chong H. Generation of an anti-tumour immune response in a non-immunogenic tumour: HSVtk killing in vivo stimulates a mononuclear cell infiltrate and a Th1-like profile of intratumoural cytokine expression. Int J Cancer 1997; 71: 267-74.

29 Ramesh R, Marrogi AJ, Munshi A, Abboud CN, Freeman SM. In vivo analysis of the 'bystander effect': a cytokine cascade. Exp Hematol 1996; 24: 829-38. 